\title{
Zum Grundsatzproblem der Berechnung und konstruktiven Gestaltung ungewöhnlicher Bauteile
}

\author{
Eberhard Wegener
}

Der Zwang zur Einhaltung von Vorschriftenwerken und die Notwendikeit, Dimensionierungsprobleme zeitlich effektiv durch Software zu lösen führen in immer stärkerem Maße dazu, nach Modellösungen für Problemstellungen zu suchen. Werden sie nicht gefunden, scheint das Problem unlösbar. Am Beispiel einer übergroßen Reinigungsöffnung für einen Festdachtank $10000 \mathrm{~m}^{3}$ wird das Problem dargestellt und die Lösung über eine ingenieurtechnische Näherung gefunden.

\section{Ausgangssituation}

Das häufig auftretende und insbesondere im der Rahmen der Ausbildung feststellbare Problem läßt sich in drei Fragen gliedern:

1. Denkweisen der Festigkeitsrechnung und der rechnerischen Erfassung von Konstruktionsdetails

2. Praktische Gegebenheiten zur Lösung einer Konstruktionsaufgabe in Hinblick auf eine sichere Tragfähigkeit

3. Die Zuverlässigkeit sogenannter exakter Lösungen und ingenieurmäßiger Näherungslösungen.

Es können zwei grundsätzlich unterschiedliche Denkweisen der Festigkeitsrechnung herausgestellt werden:

a) die Anpassung der Ausführung an vorhandene Berechnungsalgorithmen, die als gewöhnliche Berechnung bezeichnet werden kann,

b) die von den Grundlagen der Festigkeitsberechnung aus zusammengestellte und aufgebaute Berechnung, die der Konstruktion in besonderer Weise angepaßt ist, wobei es sich um Konstruktionen handelt, die nicht zu einer Klasse oder Art von Konstruktionselementen gehören.

Mit der EDV-Berechnung erfolgt eine große Aufweitung von a) und auch in einen weiten Bereich von b) hinein, d.h. ein großer Teil der Konstruktionen, die früher nach b) berechnet wurden, sind ebenfalls vollständig programmiert. Dadurch verkümmert die Fertigkeit der Ingenieure, Aufgaben nach b) zu bewältigen, d.h. in der Praxis beschränken sie sich ganz auf die Benutzung vorgegebener Formeln bis zur Verwendung von Berechnungs-Software und verlieren immer mehr neben der Fertigkeit auch den Drang, neue Konstruktionen zu schaffen.

Auch Zwingenberger /1/ bemerkt, daß mit dem für Apparatebauer zur Verfuigung stehenden Regelwerk für die
Bemessung von Bauteilen zwar die Möglichkeit besteht, „...Techniker mit weniger fundiertem „Background“ anzulernen und nach den gut aufbereiteten Rezepten auch komplizierte Apparate selbständig bemessen zu lassen“, bemerkt aber auch, daß ,hiermit aber auch eine große Gefahr verbunden ist. In den meisten Fällen fehlt die Urteilsfähigkeit über die Grenzen der dargebotenen Formeln und über die Auswirkungen kombinierter Lastfälle..."

Jedes Konstruktionsproblem wird theoretisch nur näherungsweise abgebildet. Der Näherungscharakter beruht bei der sogenannten exakten Theorie in den Abweichungen des konkreten Gegenstandes von den idealisierenden Voraussetzungen der exakten Theorie. Eine sog. exakte Lösung heißt also nicht, daß sie die Wirklichkeit genau abbildet ( was häufig angenommen wird), sondern daß die Lösung auf einen exakten mathematisch-mechanischen Ansatz bezogen ist.

Ein von den Voraussetzungen einer Lösung stärker abweichender Fall wird folglich mit immer geringerer $\mathrm{Zu}$ verlässigkeit von der Abbildung der Wirklichkeit abweichen. So kann die mathematisch-mechanische Lösung einen geringeren Näherungswert haben als eine ingenieurmäßige Näherung, wenn das vorliegende Problem in sie eingezwängt wird. Die Bewertung dieser Lösung als Ausdruck des Vorhandenen, d.h. „exakt" ist eine Täuschung.

Diese negativen Seite der modernen Entwicklung, die besonders die Konstrukteure betrifft, muß bewußt gemacht werden, weil das große Ingenieurpotential der Absolventen in diesem Prozeß immer geringere Erfahrungsmöglichkeiten der Verknüpfung von Technologie - Konstruktion und Berechnung besitzt. Dies ist gleichzusetzen mit einem fortlaufenden Verlust von Entwicklungsmöglichkeiten aus diesem Denkpotential.

Es muß daher davon ausgegangen werden, daß die Konstrukteure auch heute noch grundsätzlich jede Konstruktionsaufgabe auch in der Verknüpfung mit der Festigkeitsberechnung lösen können müssen, und daß hierbei die Verwendung vorberechneter Abmessungswerte bis zur Benutzung von Berechnungs-Software nur sinnvoll möglich ist, wo die Fähigkeit bestehen bleibt, die Aufgabe selbst auch ohne diese Hilfsmittel zu lösen.

Allerdings müssen die an die Ausbildungsinhalte von Fachhochschul- und Universitätsabsolventen unterschiedlich gestellten Anforderungen zur Beherrschung von der Beanspruchung angepaßten Berechnungs- 
verfahren und die dazu notwendigen Kenntnisse und Fertigkeiten differenziert werden. Im Zusammenhang stehende Problembehandlungen sind $u$.a. auch in $/ 2 /$ bis /4/ veröffentlicht.

Bei den praktischen Gegbenheiten sind zwei grundsätzlich unterschiedliche Methoden herauszustellen:

$\alpha$ ) die Aufgabe läßt sich nach vorhandenen Vorschriften mit oder ohne Benutzung von EDV-Programmen lösen bzw. die Lösung läßt sich aus vorhandenen Unterlagen zusammensetzen

$\beta$ ) die Konstruktion läßt sich nicht an die vorhandenen Unterlagen anpassen und muß schöpferisch für die Berechnung mit möglichen Rechenansätzen aufbereitet, d.h. es muß erst das Berechnungsmodell gefunden werden.

In die erste Situation fallen alle Aufgaben, die sich in die Bauelemente der Technischen Mechanik zerlegen lasen und aus diesen rechnerisch zusammengebaut, d.h. aneinandergesetzt werden können. Hier findet also zunächst eine Deduktion des Ganzen in seine Teile statt, die induktiv nach der Bemessung zusammengestzt, die ganze Konstruktion ergeben. Im Gegensatz dazu können Aufgaben nach $\beta$ ) dadurch gelöst werden, daß ein Modell ineinanderlaufender Bauelemente aus der Technischen Mechanik den Festkörper abbilden. Bei der Betrachtung der Zuverlässigkeit ist davon auszugehen, daß die theoretische Abbildung der Konstruktion stets nur eine Näherung darstellt und wir zwei Näherungsgrade unterscheiden können:

x) den Näherungsgrad der sog. exakten Lösung, d.h. den Grad der Erfuillung der idealisierenden Voraussetzungen, die bei der exakten Berechnung als Voraussetzungen getroffen werden

y) den Grad der sog. ingenieurmäßigen Näherungsrechnung, bei der das zusammengesetzte Konstruktionsobjekt aus berechenbaren, einfachen Bauelementen gedacht wird, wobei die Genauigkeit von der Übereinstimmung der gedachten Glieder in alleiniger Wirkung und in ihrem Zusammenwirken abhängt.

Zu den exakten Verfahren gehören Lösungen nach der Elastizitätstheorie, wobei Weiterungen in den plastischen Bereich nicht als qualitativ anders, sondern nur als Ergänzung der Randbedingungen auftreten einschließlich der modernen Verfahren zur Benutzung von finiten Elementen.

Zu den ingenieurmäßigen Näherungen gehört die Reduzierung der Bauteile in eine niedere Dimension, d.h. Tragkörper in charakteristische Tragflächen, und Tragflächen in charakteristische Tragstäbe und Seile.

Beim Zerlegen komplizierter Apparatebauteile in mehrere Einzelelemente, deren Spannungen sich relativ einfach berechnen lassen, muß jedoch beachtet werden, daß sich die Ränder dieser aneinanderstoßender Elemente unter Einwirkung der jeweiligen äußeren Lasten in der Regel ungleichmäßig deformieren und sich hier- aus Zwangsverformungen, die zum Ausgleich notwendig sind, und damit Störspannungen, bilden. Solche Störstellen sind z.B. geometrische Störstellen (schroffe Änderung von Wanddicke, Form und Krümmung) oder nicht-geometrische Störstellen (Änderung von elastischen Konstanten, Werkstoff, Temperatur, Belastung).

Das Stoff-Zeit-Problem in der Ausbildung von Ingenieuren und die zum Studienbeginn mangelnden Voraussetzungen, sich mit der Denkweise und den Methoden der Technischen Mechanik vertraut zu machen und damit die Voraussetzungen für die Übertragung dieser in das Fachstudium zu schaffen, sind eine allgemeine Erfahrung. Am Beispiel der Entwicklung einer übergroßen Reinigungsöffnung für einen $10.000 \mathrm{~m}^{3}$ Festdachtank wird die Vorgehensweise einer ingenieurmäßigen Näherung vorgestellt.

\section{Aufgabenstellung und Lösungsfindung}

Die Aufgabe besteht darin, den Mantel eines stehenden zylindrischen Tankes mit einer quadratischen Reinigungsöffnung auszurüsten, die durch ihre Abmessungen von $3500 \mathrm{~mm} \times 3500 \mathrm{~mm}$ als übergroß einzustufen ist (Bild 1). Eine Öffnung dieser Größe ist erforderlich, um für die mechanische Reinigung ein Großgerät, das in den Tank von außen einfährt, einsetzen zu können. Daraus resultiert, daß die Schwellenhöhe max. $400 \mathrm{~mm}$ betragen darf.

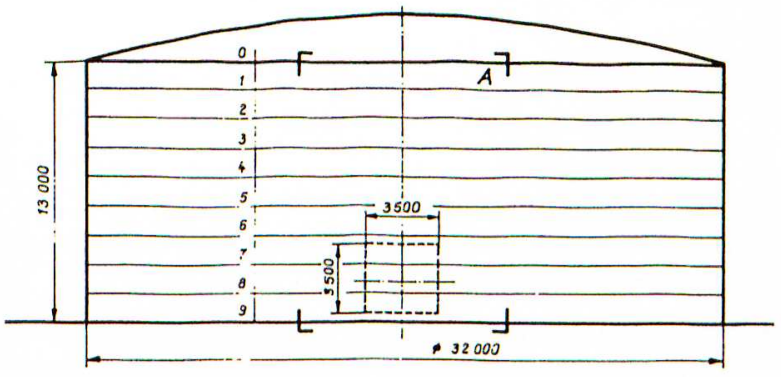

Bild 1: Festdachtank $10000 \mathrm{~m}^{3}$ mit vorzusehender übergroßer Reinigungsöffnung

Der Tank ist mit einem Durchmesser von $32.000 \mathrm{~mm}$ aus 9 Schüssen hergestellt, arbeitet bei einer Betriebstemperatur von $60^{\circ} \mathrm{C}$, drucklos, Medium Schwelwasser, Werkstoff St 38 b-2 TGL 7960 (jetzt S 235JRG2 DIN EN 10025 /5/; W.-Nr. 1.0038 ) mit Werkszeugnis TGL 050049 (DIN 50049 /6/). Das Beanspruchungsverhältnis der Stumpfschweißnähte wurde mit $v_{Z}=0,8$ berücksichtigt. Für den zeit-, dicken- und temperaturabhängigen Festigkeitskennwert gilt DIN EN 10 025. Die Ausführung erfolgte nach DIN 4119 T.1 /7/. Für die Festigkeits- und Stabilitätsrechnung ist DIN 4119 T.2 / 8/ maßgebend.

Da es sich um eine nachträgliche konstruktive Veränderung des Tankmantels handelt scheint es sinnvoll, die eventuellen Tragreserven der betroffenen Mantelschüsse zu ermitteln, um gegebenenfalls die Verschwächung durch den Ausschnitt wenigstens teilweise aufnehmen zu können. Bei der Überprüfung der ausgeführten Wanddicken wird mit einer Füllhöhe bis zum Dach- 
eckring (OK Mantel) gerechnet. Eine nochmaliger Stabilitätsnachweis für den ausgeführten Tank (/9/ - /11/) ist nicht erforderlich.

Da DIN 4119 keine verbindliche Berechnungsvorschrift darstellt, sondern lediglich darüber informiert, welche Berechnungsgrundlagen ( im wesentlichen Stahlbaustandards) angewendet werden können, kann eine Überprüfung nach BR-B 6/12/ erfolgen.

Die erforderliche Wanddicke der Schale unter hydrostatischem und inneren Überdruck im Abstand $x_{K}$ von der maximalen Fülllhöhe wird dann ermittelt zu

$s_{M}=\frac{\rho_{F} \cdot x_{K} \cdot 10^{-3}+p_{i}}{v_{Z} \cdot[\sigma]} \cdot r_{M}+c_{M}$

mit

$\mathrm{s}_{\mathrm{M}} \mathrm{mm}$ rechnerisch erforderliche Wanddicke

$r_{F} \quad \mathrm{~kg} / \mathrm{dm}^{3}$ Dichte des Füllmediums

$x_{K} \quad \mathrm{~cm} \quad$ laufende Koordinate von der max. Füllhöhe nach unten

$\mathrm{p}_{\mathrm{i}} \quad \mathrm{kp} / \mathrm{cm}^{2}$ innerer Überdruck

$v_{Z} \quad$ - Schweißnahtwertigkeit bei Zug

$\mathrm{r}_{\mathrm{M}} \quad \mathrm{mm} \quad$ Innenradius des Zylinders

$\mathrm{c}_{\mathrm{M}} \mathrm{mm} \quad$ Wanddickenzuschlag für den Mantel

[s] $\mathrm{kp} / \mathrm{cm}^{2}$ zulässige Spannung

Dabei stellt der Nenner von Gl. (1) den Berechnungsdruck nach AD-Merkblatt B 0 /13/ dar, der sich infolge fehlenden Innendruckes auf den hydrostatischen Druck vermindert, womit sich die Bemessungsgleichung zu

$s_{M}=\frac{D \cdot p}{2 \cdot \frac{K}{S} \cdot v_{Z}}+c_{M}$

mit $p=\rho_{F} \cdot g \cdot x_{K} \cdot 10^{-6}$

und

p $\quad \mathrm{N} / \mathrm{mm}^{2}$ Berechnungsdruck

D $\mathrm{mm}$ Durchmesser des Zylinders

$\mathrm{s}_{\mathrm{M}} \quad \mathrm{mm}$ rechnerisch erforderliche Wanddicke

$\mathrm{r}_{\mathrm{F}} \quad \mathrm{kg} / \mathrm{m}^{3} \quad$ Dichte des Füllmediums

$x_{F} \quad m \quad l$ laufende Koordinate von der maximalen Füllhöhe nach unten

K $\mathrm{N} / \mathrm{mm}^{2}$ Festigkeitskennwert $\mathrm{f}$ (Werkstoff,s,t)

$\mathrm{S}$ - $\quad$ Sicherheitsbeiwert

g $\mathrm{m} / \mathrm{s}^{2} \quad$ Erdbeschleunigung

vereinfacht.

Das Tragverhalten wird jedoch übersichtlicher, wenn die sich aus der hydrostatischen Druckhöhe ergebenden Ringkräfte $n_{\varphi}=p \cdot R$ auf die rechnerisch ausnutzbare Wanddicke $s_{R}=s_{\text {vor } h_{1}}-c_{M}$ (mit $c_{M}=1 \mathrm{~mm}$ Korrosionszuschlag) bezogen und die sich ergebende Ringspannung $\sigma_{K}=\frac{n_{\varphi}}{s_{R}}$ der zulässigen Ringspannung gegenuibergestellt wird. Für den ausgeführten Tank gelten die Werkstoffkennwerte nach TGL 7960, für die Schweißnähte gilt nach $/ 4 / \sigma_{z u l}=\frac{K}{S} \cdot v_{2}$.

Mit $\mathrm{K}=230 \mathrm{~N} / \mathrm{mm}^{2}, \mathrm{~S}=1,5$ und $\mathrm{v}_{\mathrm{Z}}=0,8$ ergibt sich eine zulässige Ringspannung von $\sigma_{\text {zul }}=122,6 \mathrm{~N} / \mathrm{mm}^{2}$.
In Bild 2 sind die Werte für die einzelnen Stöße für den jeweils oberen und den unteren Mantelschuß zusammengestellt. Dabei wurde - wie gewöhnlich - an den Dickensprüngen des Mantels die entlastende Wirkung des dickeren Abschnittes im Randbereich des dünneren vernachlässigt und die Schalenbiegung an dieser Stelle unberïcksichtigt gelassen. Die mittragende Breite des Mantels zum Bodenrandblech beträgt nach /14/

$b_{M}^{\prime}=0,778 \cdot \sqrt{r_{M} \cdot\left(s_{M . S t}-c_{M}\right)}$

mit $r_{M} \quad=16000 \mathrm{~mm}$ Innenradius des Zylinders

$\mathrm{s}_{\mathrm{M}, \mathrm{St}}=18 \mathrm{~mm}$ Wanddicke im Steifenbereich

$\mathrm{c}_{\mathrm{M}}=1 \mathrm{~mm}$ Korrosionszuschlag.

Die konstruktive Ausführung von $b_{M}^{\prime}=394 \mathrm{~mm}$ kann wegen der versteifenden Wirkung der noch zu entwikkelnden Ausschnittsverstärkung zugelassen werden.


Bild 2: Hydrostatischer Druck, Ringkräfte, nutzbare Wanddicke und Ringspannungsverteilung für die einzelnen Mantelschüsse 
Aus Bild 2 ist ersichtlich, daß damit an den Stößen 5 (10/ $12 \mathrm{~mm})$ und $6(12 / 14 \mathrm{~mm})$ der Tankwandung beim Nachweis $\sigma_{K x}=\frac{n_{\varphi}}{s_{R}}$ die zulässige Spannung bereits im normalen Betriebszustand uiberschritten wird. Diese theoretische Überschreitung der zulässigen Spannungsgröße ergibt sich aus dem vorgenannten üblichen Berechnungsansatz der unabhängigen Tragwirkung der Einzelschïsse. Die Überbeanspruchung an den unteren Rändern der Schuisse 5 und 6 muß durch die Tragreserve der dickeren, darunterliegenden Schüsse abgebaut werden. Dieser Spannungsabbau wird durch standardisierte Berechnungsansätze nicht berücksichtigt.

Folgende Vorgehensweisen sind möglich:

Nach /15/ ist das Problem der Dickenabstufung über der durch Momente und Querkräfte zentralsymmetrisch gestörten Membranzustand lösbar. Diese Störgrößen verursachen eine Reduzierung der Normalkräfte $n_{\varphi_{0}}$ für den oberen Schuß. Im Ergebnis kann der statische Effekt ohne Einschränkung entweder zu einer Reduzierung der Wanddicke des oberen Schusses oder bei Beibehaltung dieser zu einer Verlegung der Stoßstelle um einen Betrag $x_{i}$ in Richtung des zunehmenden hydrostatischen Druckes führen (diese Verlegung liegt bei dem Tankradius von $16 \mathrm{~m}$ etwa bei $35 \mathrm{~cm}$ ). Die angegebene transzendente Gleichung gestattet als Lösungsweg nur eine iterative Näherung.

Eine einfachere Lösung enthält /14/: aus dem Modell für die rotationssymmetrische Belastung (Bild 3) kann die größte Ringspannung des dünneren Abschnittes im Schnitt 1 bei Innendruck näherungsweise mit

$\sigma_{\varphi 1}=\begin{gathered}-p_{r 1} \cdot R \\ h_{1}\end{gathered}$

oder aus der Belastung des Mittragenden Bereiches zu

$\sigma_{\varphi}=\frac{-p_{r s} \cdot R \cdot\left(L_{c 1}+L_{c 2}\right)}{L_{c 1} \cdot h_{1}+L_{c 2} \cdot h_{2}}$

ermittelt werden, wobei die größere der beiden Spannungen maßgenbend ist.

\section{Hierin sind}

$\sigma_{\varphi} \quad \mathrm{N} / \mathrm{mm}^{2}$ Ringspannung im Kreiszylinder $\left(\sigma_{K x}\right)$

p $\mathrm{N} / \mathrm{mm}^{2}$ hydrostatischer Druck

$\mathrm{R} \quad \mathrm{mm} \quad$ Zylinderradius

h $\mathrm{mm} \quad$ Wanddicke $\left(\mathrm{s}_{\mathrm{R}}=\mathrm{s}_{\text {vorh }}-\mathrm{c}_{\mathrm{M}}\right)$

$\mathrm{L}_{c} \quad \mathrm{~mm} \quad$ charakteristische Länge des Schalenelementes, mittragende Breite unter Berüicksichtigung der am Rand auftretenden zusätzlichen Biegekräfte

$$
L_{c}=\frac{1}{\lambda}=0,78 \cdot \sqrt{R \cdot h}
$$

Indices:

$r$ radial

$x \quad$ laufende Koordinate von oben nach unten

$\mathrm{s}$ auf den Schwerpunkt des mittragenden Bereiches bezogen.



Bild 3: Modell für die rotationssymmetrische Belastung des abgestuften Zylinders unter hydrostatischem Druck /14/

Die größte Biegespannung beträgt dann

$\sigma_{b} \approx \pm 1,82 \cdot R \cdot\left(\frac{p_{r 1}}{h_{1}}-\frac{p_{r 2}}{h_{2}}\right)$

Mit $\mathrm{h}_{1}=9 \mathrm{~mm}, \mathrm{~h}_{2}=11 \mathrm{~mm}$ und $\mathrm{R}=16000 \mathrm{~mm}$ ergeben sich die Werte für die Stöße 5 und 6 nach Tab.1.

\begin{tabular}{|c|c|c|c|c|c|c|c|c|}
\hline Sto $B$ & $\begin{array}{l}\mathrm{L}_{\mathrm{c1}} \\
\mathrm{mm}\end{array}$ & $\begin{array}{l}\mathrm{L}_{\mathrm{c} 2} \\
\mathrm{~mm}\end{array}$ & $\begin{array}{l}\rho_{x 1} \\
\mathrm{~N} / \mathrm{mm}^{2}\end{array}$ & $\begin{array}{l}\mathrm{P}_{\times 2} \\
\mathrm{~N} / \mathrm{mm}^{2}\end{array}$ & $\begin{array}{l}\mathrm{ps}_{\mathrm{s}} \\
\mathrm{N} / \mathrm{mm}^{2}\end{array}$ & $\underset{\mathrm{N} / \mathrm{mm}^{2}}{\sigma_{k x}(1 / 2)\left(\sigma_{\varphi 1}\right)}$ & $\begin{array}{l}\sigma_{k^{*}}\left(\sigma_{Q}\right. \\
\mathrm{N} / \mathrm{mm}^{2}\end{array}$ & $\begin{array}{c}\sigma_{b} \\
\mathrm{~N} / \mathrm{mm}^{2}\end{array}$ \\
\hline 5 & 296 & 327 & 0,06734 & 0,0734 & 0,07096 & $119,7 / 106,8$ & 112,97 & 23,57 \\
\hline 6 & 327 & 355 & 0,08136 & 0,08804 & 0,0852 & $118,3 / 108,6$ & 113,2 & 18,17 \\
\hline
\end{tabular}

Tabelle 1: Mittragende Breite, hydrost. Druck, Ringspannungen und Biegespannungen an den Stößen 5 und 6

Der Tankmantel ist daher optimal ausgelastet und besitzt keine ausreichenden Tragreserven zur Aufnahme eines Ausschnittes. Der Ausschnitt muß also verstärkt werden. Bild 4 zeigt den vergleichmäßigten Spannungsverlauf.

Naturgemäß wird nun versucht, eine Vorschrift zur Berechnung einer solchen Ausschnittsverstärkung zu ermitteln. DIN 4119 T2 /8/ legt nur fest: „werden kreisförmig Mannlöcher und Stutzen über dem Boden angeordnet, so kann auf einen Spannungsnachweis verzichtet werden, wenn die ausgefallene Fläche durch eine Ersatzfläche $\left(A_{E}\right)$ ersetzt wird, die aus Teilen mit gleicher (oder höherer) Festigkeitskennwerten besteht". Diese Methode ist auch den Vorschriften z.B. TGL 32 903/15 /16/, BR-A 15/17/ oder AD-Merkblatt B $9 / 18 /$ gemeinsam. Generell ist damit die Dimensionierung kreisförmiger Ausschnittsverstärkungen möglich und näherungsweise elliptischer oder Langlochausschnitte. Auch neuere Untersuchungen /19/, /20/ beziehen sich auf kreisförmige Querschnitte bei Ausschnitten. Bei dünnwandigen Großbehältern wird gewöhnlich angenommen, daß die Summe der Membrankräfte des halben Ausschnittsdurchmessers r gem. Bild $5 / 14$ / durch den tragenden Ausschnittsrand (ggf. unter Zuhilfenahme eines Verstärkungsbleches) von der Breite $b=r$ gleichmäßig verteilt seitlich am Stutzen vorbeigefuihrt wird. 


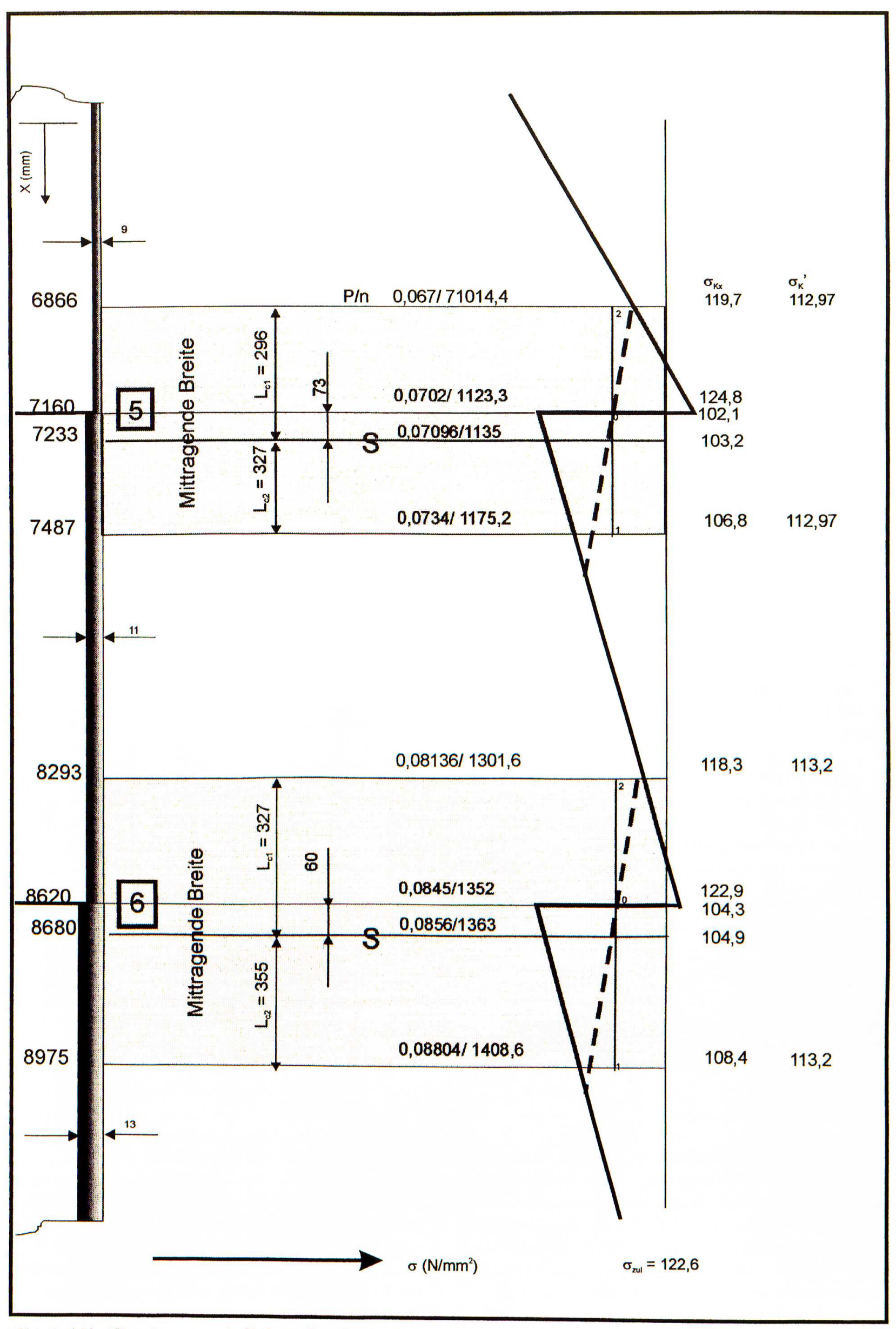

Bild 4: Vergleichmäßigter Spannungsverlauf in den Stoßbereichen 5 und 6 


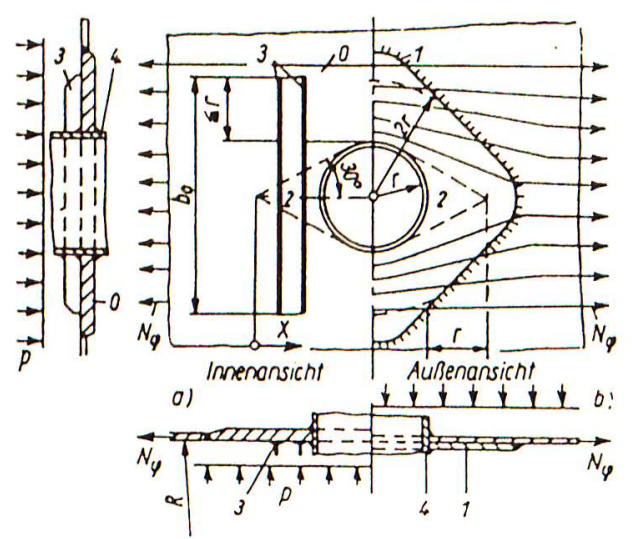

Bild 5: Ausschnittsverstärkung nach Drescher /14/

/21/ enthält auch die Festlegung, daß bei gleichen Werkstoffen der ausfallende Querschnitt $A_{a}$ zu ersetzen ist (Bild 6) durch

$A_{a} \leq A=A_{M}+A_{V}+A_{R}$

mit $A_{M}=$ Unterschied zwischen rechnerischer und vorhandener Wanddicke x Ausschnittsdurchmesser

$A_{V}=\quad$ Breits $x$ Dicke des Verstärkungsbleches

$A_{R}=A_{1}+\frac{A_{2}}{4} b z w \cdot A_{1}+0,55 \cdot \sqrt{d_{S} t \cdot t_{S t}} \cdot t_{S t}$,

für $A_{R}$ ist der kleinere Wert maßgebend

$A_{1}=$ symmetrisch zur Mittelebene gelegene Stutzenfläche

$A_{2}=$ restliche Stutzenfläche

$\mathrm{d}_{\mathrm{St}}=$ Mittlerer Stutzendurchmesser

$\mathrm{t}_{\mathrm{St}}=$ Wanddicke des Stutzens

Die Anordnung einer derartig großen Fläche $\left(A_{A}\right)$ ist bei den gegebenen Wanddicken des Tankmantels und der Ausschnittslage selbst nicht realisierbar. Es ist demzufolge eine ingenieurtechnische Näherung, wie im Teil 1 beschrieben, zu entwickeln, Grundlagen hierzu enthält $/ 14 /$.

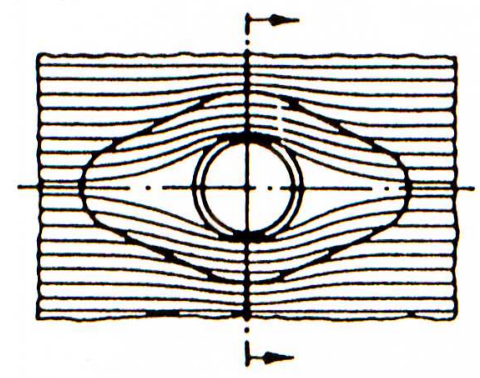

Bild 6: Ausschnittsverstärkung nach /21/

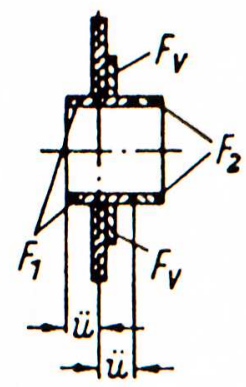

Zur Abtragung der Last aus dem $N_{\varphi}$-freien Bereich nach den Seiten wirkt das Moment $M_{x}=3 \cdot p \cdot x^{2} / 8$, dessen Aufnahme die Verstärkung des Mantelbleches bei dem vorhandenen großen Ausschnitt durch Aussteifungsrippen erfordert (Bild 7), die Ausschnittsverstärkung wird ebenfalls im Bereich $b \leq L_{c}$ am Ausschnittsrand konzentriert. Die Randscheibe mit der Höhe $\approx 0,7 \cdot d$ leitet die Ringkräfte $d \cdot N_{\varphi}=2 \cdot Z$ zu den Randverstärkungen. Sie wird durch Rippen, die zugleich die Plattenwirkung im $N_{\varphi}$-freien Bereich sicherstellen, ausgesteift. Die konstruktive Umsetzung wird im Teil 3 beschrieben.

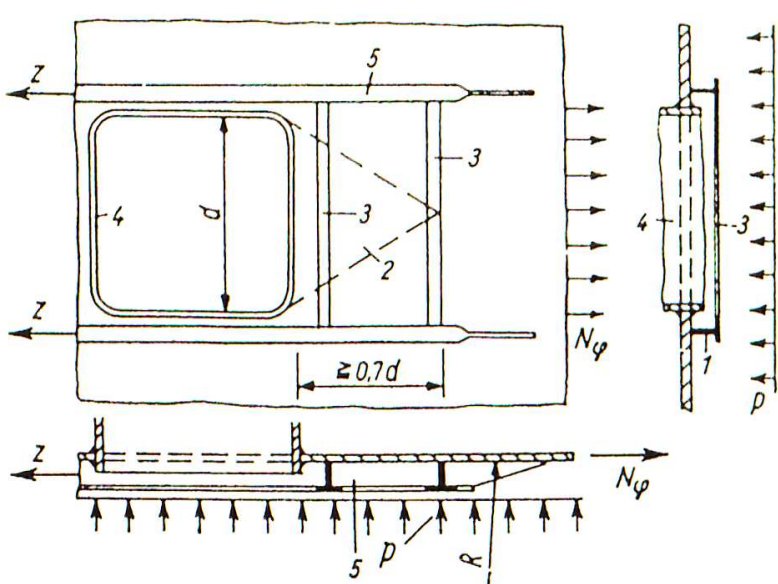

Bild 7: Modell der Ausschnittsverstärkung über Zuganken und Plattenaussteifung /14/

\section{Konstruktive Lösung und Realisierung}

Die im Teil 2 Bild 7 dargestellte Lösungsvariante wurde gemäß Bild 8 entwickelt, indem das theoretische Bild des Tragmechanismus in die klassischen Tragelemente (Stab, Platte, Scheibe, Schale) zerlegt und nach den klassischen Regeln bestimmt wird.

\section{Theoretisches Systembild der Ausschnittskonstruktion}

Das Bild des statischen Systems der Ausschnittskonstruktion wird aus folgender Überlegung abgeleitet:

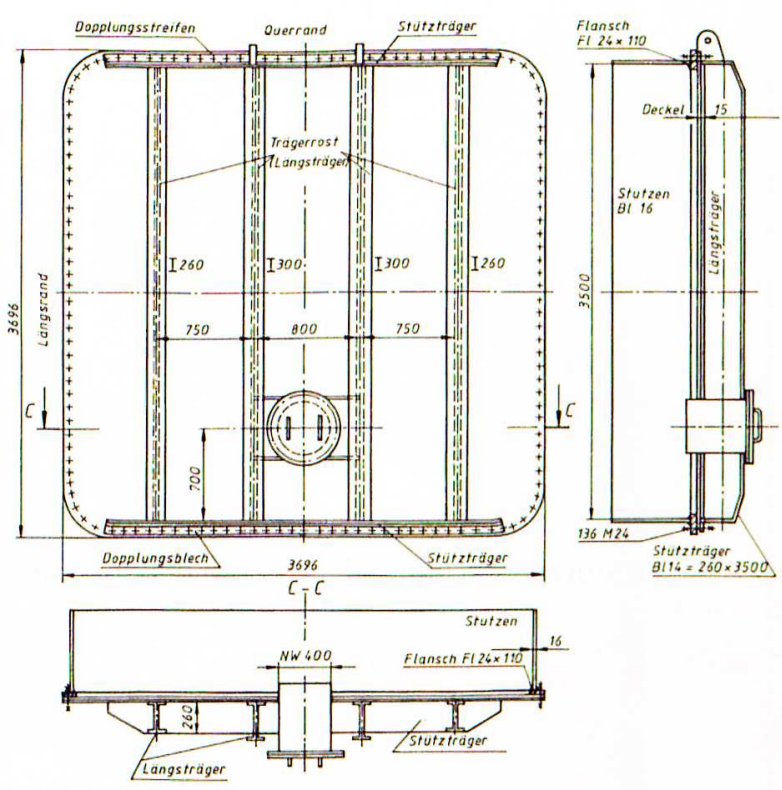

Bild 8: Konstruktiver Entwurf der quadratischen, übergroßen Reinigungsöffnung (Ausschnittsverstärkung - Umleitungskonstruktion)

Nach Bild 8 schneidet die Öffnung den Mantel auf eine Länge von $3532 \mathrm{~mm}$, beginnend etwa $400 \mathrm{~mm}$ über dem Bodenblech (also im Bereich der größten Belastung) auf. Die durch die Durchschneidung freiwerdenden Mantelringkräfte ( $\mathrm{N}=6,02 \mathrm{MN}$ ), die mit dem hydrostatischen Druck im Gleichgewicht stehen, bauen sich innerhalb der Längsrandzone von ihrem Normalwert bis auf den Wert Null am freien Ende ab. Sie müssen deshalb im Breich der Längsrandzone aufge- 
fangen und zu den Rändern abgeleitet werden, um an diesen über Zugbänder wieder miteinander verkoppelt zu werden. Da die Mantelringkräfte im Bereich der Längsrandzonen nicht mehr voll wirksam werden, kann innerhalb dieser Bereiche der hydrostatische Druck auch nicht von ihnen aufgenommen werden. Es liegt nahe, die Wirkung der Mantelringkräfte zur Lastaufnahme innerhalb der Längsbereiche ganz zu vernachlässigen und die Längsbereiche als leicht gekrümmte Plattenscheibe aufzufassen, wobei die angeschlossenen Mantelringräfte von der Scheibenwirkung, der hydrostatische Druck innerhalb dieses Bereiches von der Plattenwirkung aufgenommen und abgetragen werden. Sind die Längsrandbereiche als Plattenscheibe für diese Belastung bemessen und die Zugbänder für deren Stützkräfte ausgelegt, dann ist die Tragfähigkeit in diesem Bereich sichergestellt.

Die Zuggurte, die die Stuitzkräfte der beiden Längsrandzonen aufnehmen und gegenseitig aufheben, folgen dem Verlauf der Mantelkrümmung. Dabei haben sie die Tendenz, sich geradezustrecken, es sei denn, ihr gekrümmter Verlauf bildet zugleich die Stützlinie einer Querbelastung aus dem hydrostatischen Druck. Diese Querbelastung liefert die Stuitzkräfte der Plattenwirkung der Längsrandzone dee Öffnungsverschlusses. Der hydrostatische Druck auf den Öffnungsdeckel kann an den Längsrändern nicht aufgenommen werden. Er muß ganz zu den Querrändern und von dort aus in die Zuggurte übergeleitet werden. Die Überleitung erfolgt über die Stutzenbleche an den Querrändern. Als Besonderheit der Stutzenabschnitte der Längsränder ist zu beachten, daß sie den hydrostatischen Druck aus den anschließenden Endfeldern sowohl des Deckels als auch der Plattenwirkung der Plattenscheiben aufzunehmen und an die Stutzenabschnitte der Querränder abzugeben haben, damit sie von dort in die Zuggurte uibergeleitet werden können. Die Besonderheit dieser Überleitung besteht darin, daß das Stutzenblech um die halbe Breite der Zuggurte aus der Zuggurtachse versetzt ist und bei der Überleitung der Kräfte aus dem Stutzen ein Versetzungsmoment auftritt, das im Zusammenwirken der Zuggurte durch das anschließende Stutzenblech aufgenommen werden muß. Zur Vergleichmäßigung der Überleitung werden die Stutzenabschnitte der Querränder über ihre Enden hinaus um 1650 mm vorgebunden. Der hydrostatische Druck im Bereich der Querränder (Bauhöhe der Zuggurte) wird von dem Mantelblech in diesem Bereich aufgenommen. Werden die Zuggurte für diese Belastung bemessen, dann ist auch im Querrandbereich die Tragfähigkeit sichergestellt.

\section{Konstruktive Gestaltung der Ausschnittskonstruktion}

Die erläuterte Umleitungskonstruktion besteht aus 2 Plattenscheiben an den Längsrandzonen des Mantelbleches (Bl 14 bis Bl 18) und einem in diesem Bereich aufgelegten Dopplungsblech (Bl 14), sowie vertikel angeordneten, in Schweißkonstruktion ausgefuihrten Tförmigen Rippen aus Bl 14. Sowohl die Platte als auch die Scheibe sind an ihren Horizontalrändern gestiitzt und leiten die Stiitzkräfte in die Zuggurte aus BI 30x250x6630 mm aus S355J2G3 DIN EN 10025 ein, die so kompakt und so dicht wie möglich an den Querrändern angeordnet sind.

Der Ausschnitt wird durch einen ebenen Deckel verschlossen. Er ist an dem quadratischen Stutzen mithilfe eines Flansches angeschraubt. Das Deckelblech besteht aus S235JRG2 DIN EN 10025 und besitzt die Abmessungen BI 16×3700×3700 mm. Im Ausschnittsdeckel befindet sich noch eine Arbeitsöffnung DN 400, die nach den üblichen Regeln der Technik ausgebildet ist. Der Deckel wird durch einen ebenen Rippenrost, der keine Zwischen-Querrippen besitzt, ausgesteift. Auch hier ist eine zusammengesetzte Bauweise erforderlich. Die mögliche Ausfuihrung als ebene Platte, wie sie nach /22/ bzw. /23/ möglich wäre, liefert eine nicht realisierbare Plattenstärke. Die Roststützung erfolgt an den Querrändern, wodurch die für die Umleitung der Ringkräfte erforderliche Lasteinleitung aus dem Deckel gewährleistet wird. Die Stuitzträger des Rostes sind aus Bl 14×260×3520 mm, an sie schließen sich 4 Längsträger aus Doppel-T 300 bzw. 260 aus S355J2G3 DIN EN 10 025 an. Der hydrostatische Druck auf den Deckel wird uiber das Deckelblech geführt und zunächst von den Längsträgern und - aus den Endfeldern - an den Flanschrändern aufgenommen. Die Einzelstuitzkräfte der Längsträger werden durch die Stützträger vergleichmäßigt und über deren Anschluß an das Deckelblech in die Querränder und von dort aus über die Flanschquerränder und die Stutzenquerränder in die Zuggurte geleitet. Die Stützung des Deckels erfolgt so überwiegend an den Querrändern.

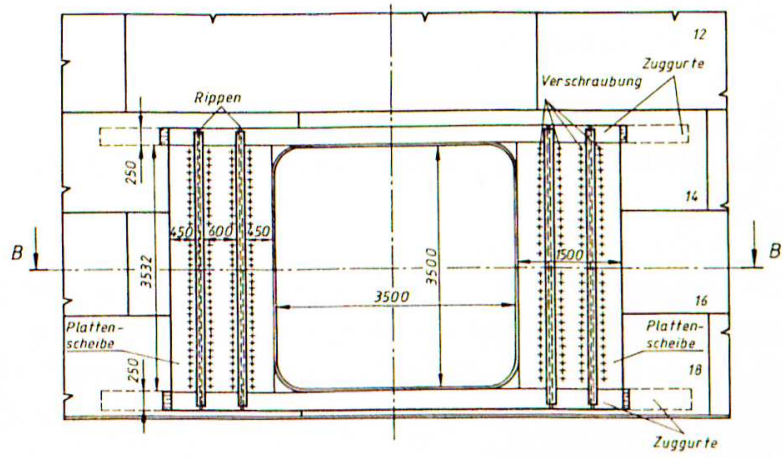

$B-B$

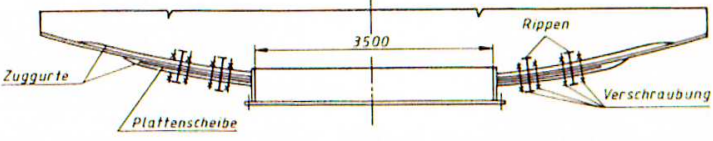

Bild 9: Stutzen- und Deckelausführung

Die Lastübertragung vom Deckel in den Stutzen erfolgt nach Bild 9 über einen geschraubten Flanschanschluß Bl 24x110. Bei der Berechnung wird die unterschiedliche Belastung auf die Querränder und die Flanschlängsträger berücksichtigt. Die höhere Belastung der Querränder erfordert eine Verstärkung der Deckelquerränder durch je einen aufgelegten Dopplungsstreifen von $\mathrm{Bl}$ 20x50×3620 mm. Auf eine mögliche Ausfuihrung der Flanschlängsränder mit einer geringeren Dicke und einer geringeren Anzahl Schrauben wird verzichtet. 


\section{Berechnung der Plattenscheibe an den Längsrändern sowie der Zuggurte an der Querrandzone der Öffnung}

Die Ringkräfte werden von einer randverstärkten Scheibe, bestehend aus dem mittragenden Stutzenblech und dem verstärkten Mantelblech, aufgenommen und zu den Zuggurten abgeleitet. Die Zuggurte nehmen die Stiitzkräfte dieser als vertikaler Balken wirkenden Scheibe auf. Zur Stabilisierung in radialer Richtung werden jeweils 4 Rippen T-förmig innen und außen angeordnet, die auf das verstärkte Mantelblech aufgeschweißt werden. Aufgrund der Abmessungen wird die Verstärkung des Mantels durch das Dopplungsblech Bl 14 neben den Rippen mit dem Mantelblech zur Gewährleistung des Zusammenhanges verschraubt. Die anteilige Ringkraft wird durch die Kehlnaht in das Dopplungsblech übergeleitet.

Für den Nachweis der Scheibenwirkung gelten folgende Überlegungen: Die Ringspannungen müssen zu den Querrändern geleitet werden. Sie beanspruchen die in Kraftrichtung liegende Längsrandzone der Öffnung auf Biegung. Der Biegequerschnitt der Scheibe wird durch das Mantelblech, einen mittragenden Abschnitt des Stutzenbleches und das auf die Behälterwand aufgesetzte Dopplungsblech gebildet. Als Profilhöhe wird ein ringspannungsfreier Bereich von $0,7 \cdot d=2450 \mathrm{~mm}$ angenommen. Am Stutzenblech und am Mantel sowie an den Schweißnähten wird ein Korrosionszuschlag von $1 \mathrm{~mm}$ beruicksichtigt. Der rechnerische Nachweis wurde erbracht für

- die Biegespannungen an der angenommenen oberen und unteren Begrenzung des Biegequerschnittes

- die Schubspannung im Steg und in den Schweißnähten

- den Anschluß der anteiligen Ringkraft in das Dopplungsblech durch die Kehlnaht (Schub- und Biegespannungen).

Die Verschraubung beteiligt sich an der Überleitung der anteiligen Ringkraft in das Dopplungsblech, wird aber rechnerisch nicht beriicksichtigt. Der Beulnachweis für die Druckzone des Steges entfällt wegen der Aussteifung durch die Rippen.

Für den Nachweis der Plattenwirkung gilt: Die Randzone des Ausschnittes wird aufgrund der fehlenden Membranwirkung auf Biegung beansprucht. Es werden deshalb zur Aussteifung des vorhandenen Querschnittes jeweils Rippen innen und außen angeordnet. Die Profile werden für eine Belastungsbreite von $600 \mathrm{~mm}$ und eine mittlere radiale Belastung von $0,1085 \mathrm{MPa}$ bemesen. Sie liegen und außen auf den Zuggurten auf und sind innen an das Mantelblech und außen an das Dopplungsblech mit Kehlnähten angeschlossen. Der rechnerische Nachweis erstreckt sich auf den Nachweis der zulässigen Biegespannung der Schrauben sowie der Überleitung der Stuitzkräfte der Rippen auf die Zuggurte. Die Schubkraft in der geschraubten Fuge der Rippen wird auf die Schraubenanzahl auf der halben Länge der Rippen gleichmäßig verteilt angenommen. Ein Nachweis des Mantelbleches durch die Schrauben- schwächung ist nicht notwendig, weil im Schnitt durch die Schraubenlinie die Mantelkraft durch den Anschluß an das Dopplungsblech bereits auf die Hälfte verringert wird.

Entsprechend der konstruktiven Gestaltung sind die Ränder des Ausschnittes an der Querrandzone innen und außen durch aufgesetzte Flacheisen verstärkt. Diese sind unter der Annahme dimensioniert, daß sie die Mantelzugkräfte aus dem Bereich der Reinigungsöffnung allein aufnehmen. Die durchgeschnittene Ringkraft war zu gleichen Teilen dem Dopplungsblech und dem Mantelblech zugeordnet worden. Entsprechend erfolgt der Nachweis des Anschlusses der Plattenscheibe an die Zuggurte. Die Zuggurte werden an ihren Enden verjüngt und im vorgeschriebenen Bereich umlaufen mit einer Kehlnaht angeschlossen.

\section{Beurteilung der Lösung und ihre Ausführung}

Die Bemessung der Umleitungskonstruktion nach der einfachen Tragfähigkeitstheorie gestattete die Entscheidung, daß aufgrund ausreichender Übereinstimmung der idealisierten theoretischen Bilder mit dem wirklichen Tragverhalten die komplizierte erstmalige Ausfuihrung ohne Versuch vorgenommen werden konnte. Ein solcher Versuch wäre jedoch vorzunehmen, wenn die dargestellte Lösung - als Sonderlösung des Problems zu einer Standardlösung verallgemeinert werden soll. In diesem Fall ist der Grad der Übereinstimmung des Bildes mit dem Tragverhalten im Versuch nachzuweisen mit dem Ziel, Tragreserven aufzuspüren.

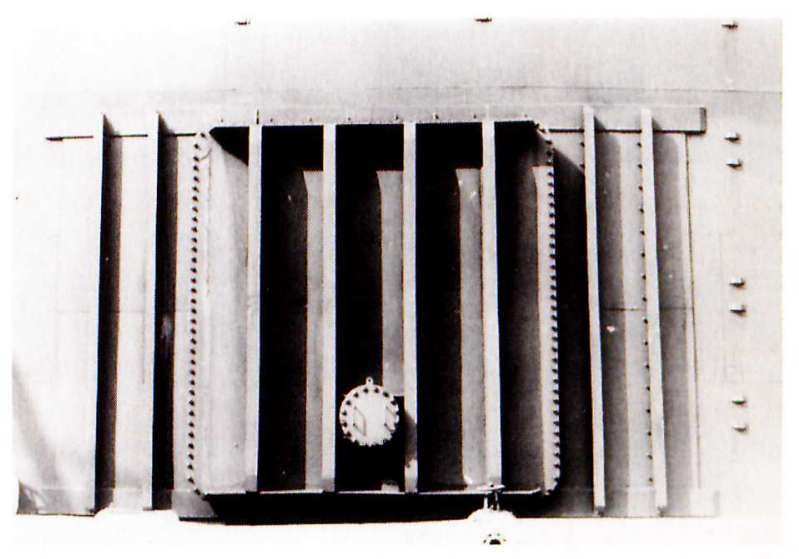

Bild 10: Ausgeführte Reinigungsöffnung, Ausschnittsverstärkung und Deckelkonstruktion

Die vorgesehene Ausfuihrung wurde an einem Festdachtank $10000 \mathrm{~m}^{3}$ realisiert (Bild 10). Bis auf anfängliche Undichtheiten geringfügiger Art an der linken Schraubenreihe der Dopplung gab es keine Dichtheitsprobleme im Prüf- und im Betriebszustand. 


\section{Literatur}

[1] Zwingenberger, L.: Berechnung und Konstruktion von Apparateelementen unter Berücksichtigung moderner Berechnungsverfahren. In: Vortragsveröffentlichungen 265, 53-62, Haus der Technik Essen

[2] Krägeloh, K., Oswald, H.: Spannungen und zulässige Beanspruchung. In: Konstruktion 21(1969)12, S. 489-494

[3] Wellinger, K., Kußmaul, K., Krägeloh, E.: Verfahren zur Beurteilung der Festigkeit von Apparatebauteilen. In: Chem.-Ing.-Techn. 45(1973)11, S. 753-759

[4] Klapp, E.: Grenzen heutiger Methoden zur Berechnung der Bauteil- und Apparatefestigkeit und mögliche Erweiterungen. In: Chem.-Ing.-Techn. 46(1974)10, S. 405-411

[5] DIN EN 10025 (3.94) Warmgewalzte Erzeugnisse aus unlegierten Baustählen, Technische Lieferbedingungen. Deutsche Fassung EN 10 025: 1990

[6] DIN 50049 (4.92) Metallische Erzeugnisse. Arten von Prüfbescheinigungen. Deutsche Fassung EN 10 204: 1991

[7] DIN 4119 Teil 1 (6.79) Oberirdische zylindrische Tankbauwerke aus metallischen Werkstoffen. Grundlagen, Ausführungen, Prüfungen

[8] DIN 4119 Teil 2 (2.80) Oberirdische zylindrische Tankbauwerke aus metallischen Werkstoffen. Berechnung

[9] DIN 18800 Teil 4 (11.90) Stahlbau. Stabilitätsfälle, Schalenbeulen

[10] DASt Richtlinie 013: Beulsicherheit von Schalen. Deutscher Ausschuß für Stahlbau, Juli 1980

[11] DASt Richtlinie 017: Beulsicherheitsnachweise für Schalen - spezielle Fälle. Deutscher Ausschuß für Stahlbau, Entwurf August 1992

[12] Müller, K.: Behälter und Apparate, Tanks mit festem Dach, BR-B6. In: Richtlinienkatalog Festigkeitsberechnungen Apparate und Behälter, VEB Komplette Chemieanlagen Dresden 1975

[13] AD-Merkblatt B 0: Berechnung von Druckbehältern. Hrsg. VdTüV e.V. Essen: AD-Merkblätter. Beuth-Verlag Berlin

[14] Drescher, G.: Stählerne Großbehälter: Sonderdruck aus Ingenieurtaschenbuch Bauwesen, Band II Ingenieurbau, Teil 2. Entwurf und Ausführung: Verlag für Bauwesen 1968, Hrsg. G. Bürgermeister

[15] Thiele, R., Kupfer, W.: Bemessung abgestufter zylindrischer Tanks unter Beachtung der Randeinspannung der Teilschalen. In: Bauplanung - Bautechnik 30(1976)8, S. 394-397

[16] TGL 32 903/15 (4.89) Behälter und Apparate. Festigkeitsberechnung, Ausschnittsverstärkungen

[17] Kiinzel, G., et.al.: Behälter und Apparate. Apparatebauelemente. Ausschnitte und Ausschnittsverstärkungen, BRA 15. In: Richtlinienkatalog Festigkeitsberechnungen Apparate und Behälter, Forschungszentrum Chemieanlagen Dresden 1973

[18] AD-Merkblatt B 9: Ausschnitte in Zylindern, Kegeln und Kugeln. Hrsg. VdTÜV e.V. Essen: AD-Merkblätter. BeuthVerlag Berlin

[19] Weiß, E.Joost, H.: Zum Tragfähigkeitsnachweis von Rohrstutzenanschlüissen in Behälterwandungen. In: Chemie- Ingenieur-Technik 68(1996)1 +2, S. 119-123

[20] Weiß, E., Lietzmann, A., Rudolph, J.: Ausschnittsgrößen in Druckbehältern ohne Auswirkung auf deren Tragfähigkeit. In: Konstruktion 47(1995), S. 233-236
[21] Wedler, B.: Berechnungsgrundlagen für Bauten, 25. Auflage, Abschn. 10.4 Stahlbehälter. Verlag von Wilhelm Ernst \& Sohn. Berlin - München - Düsseldorf, 1974

[22] TGL 32903/10 (6.81) Behälter und Apparate: Festigkeitsberechnung. Sonderformen ebener Böden und Deckel

[23] Rabisch, W., et.al.: Rechteckige Bauelemente, BR-A12. In: Richtlinienkatalog Festigkeitsberechnungen, Apparate und Behälter, Apparateelemente. Forschungszentrum Chemieanlagen Dresden, 1973

\section{Autor}

Prof. Dr.-Ing. Eberhard Wegener

Technische Fachhochschule Wildau Fachbereich Ingenieurwesen/ Wirtschaftsingenieurwesen Tel. (0 33 75) 508-169 